\title{
Beyond Smith's rule: an optimal dynamic index rule for single machine stochastic scheduling with convex holding costs
}

\author{
José Niño-Mora*
}

November 12, 2000

\footnotetext{
${ }^{*}$ Dept. of Economics and Business, Universitat Pompeu Fabra, E-08005 Barcelona, Spain. E-mail: ninomora@upf.es. Web: www.econ.upf.es/ ninomora.
} 


\begin{abstract}
Most research on single machine scheduling has assumed the linearity of job holding costs, which is arguably not appropriate in some applications. This motivates our study of a model for scheduling $n$ classes of stochastic jobs on a single machine, with the objective of minimizing the total expected holding cost (discounted or undiscounted). We allow general holding cost rates that are separable, nondecreasing and convex on the number of jobs in each class. We formulate the problem as a linear program over a certain greedoid polytope, and establish that it is solved optimally by a dynamic (priority) index rule, which extends the classical Smith's rule (1956) for the linear case. Unlike Smith's indices, defined for each class, our new indices are defined for each extended class, consisting of a class and a number of jobs in that class, and yield an optimal dynamic index rule: work at each time on a job whose current extended class has larger index. We further show that the indices possess a decomposition property, as they are computed separately for each class, and interpret them in economic terms as marginal expected cost rate reductions per unit of expected processing time. We establish the results by deploying a methodology recently introduced by us [J. Niño-Mora (1999). "Restless bandits, partial conservation laws, and indexability." Forthcoming in Advances in Applied Probability Vol. 33 No. $1,2001]$, based on the satisfaction by performance measures of partial conservation laws $(P C L)$ (which extend the generalized conservation laws of Bertsimas and Niño-Mora (1996)): PCL provide a polyhedral framework for establishing the optimality of index policies with special structure in scheduling problems under admissible objectives, which we apply to the model of concern.
\end{abstract}

Keywords: stochastic scheduling, dynamic index rule, decomposition, convex holding costs, conservation laws, achievable region, linear programming relaxation, polyhedral methods.

Journal of Economic Literature Classification: C60, C61. 


\section{Introduction}

\section{Motivation and background}

Most research on single machine scheduling (both deterministic and stochastic) has addressed problems where the objective to be minimized is the total linearly weighted job flowtime. Such traditional objective assumes that jobs incur linear holding costs during their stay in the system, at rates which depend on their class (jobs in a class have the same features). The attention accorded to the linear case has been partly motivated by the relative analytical tractability of the resulting models: for a variety of such problems, researchers have established the optimality of simple scheduling rules of (priority) index type: an index is attached to each job class; jobs with larger indices are then assigned higher processing priority.

The first example of such an optimal index rule was Smith's rule [18]. This concerns the problem of sequencing a given number of deterministic jobs belonging to $n$ classes on a single machine: class $i$ jobs' processing time is $p_{i}>0$, and they incur linear holding costs at a rate of $C_{i} \geq 0$ per job per unit time in system, for $1 \leq i \leq n$. Smith's rule is based on defining the index $\gamma_{i}=C_{i} / p_{i}$ for each class $i$, and then processing first jobs whose class has larger index.

The optimality of index rules under linear holding costs has been extended to a variety of more complex single machine scheduling models. These include the extension of Smith's model to incorporate random processing times [16], scheduling multiclass queues $[2,8,7,19]$, multiarmed bandits $[5,6]$, and branching bandits $[10,22]$, which encompass all the previous models.

The traditional linear holding costs assumption, however, may appear unrealistic in practice, as argued in [20]. Research on scheduling models that incorporate nonlinear delay costs, though, is much scarcer. In the few studies along those lines, researchers typically incorporate holding costs as delay cost functions on job flowtimes. thus adopting a view of delay costs based on individual jobs. See, e.g., [14, 21, 20].

In contrast, we shall consider in this paper explicit nonlinear holding cost rates based on the congestion level from each class, which corresponds to a system-wide view of congestion costs.

In fact, it has been argued in $[17,15]$ that the search for optimal priority index rules under nonlinear delay objectives is doomed to failure, as (see [15])

there are exactly two classes of delay cost functions for which policies that are determined by time-invariant priority indices are capable of producing optimal sequences: linear delay costs and discounted linear delay costs. We consider index-based policies that allow dynamic recalculation of priority indices and show that this class does not enlarge the set of delay cost functions for which indexbased rules can produce optimal policies.

Such negative result, however, is tailored to the case where congestion costs are incorporated as delay cost functions, and not, as we do in this paper, as nonlinear holding cost rates 
on congestion levels: for the latter case we shall present in what follows an optimal dynamic priority index rule, where the indices depend both on a job's class and on the number of jobs present in that class.

\section{Problem description and formulation}

We next describe the model of concern in this paper. A single machine is available to process jobs belonging to $n$ classes, labeled $i \in N=\{1, \ldots, n\}$. Class $i$ jobs are described as follows.

Processing time: the processing time of a class $i$ job is modeled as a random variable $\xi_{i}>0$ with mean $p_{i}=E\left[\xi_{i}\right]$, variance $\sigma_{i}^{2}=\operatorname{Var}\left[\xi_{i}\right]<\infty$, and Laplace transform $\phi_{i}(\alpha)=E\left[e^{-\alpha \xi_{i}}\right]$. We assume the processing times of different jobs to be independent.

Holding cost: they incur holding costs at a rate of $C_{\left(i, m_{i}\right)} \geq 0$ units per unit time when there are $m_{i}$ such jobs in system (waiting or being processed), with $C_{(i, 0)}=0$. Costs are continuously discounted in time by factor $\alpha \geq 0$ (with $\alpha=0$ corresponding to the undiscounted case).

Initial number: there are initially $m_{i}^{0} \geq 1$ class $i$ jobs in the system.

The problem of concern is to find a scheduling policy, prescribing how to sequence the jobs for processing, within the class of admissible policies, consisting of all nonanticipative (decisions cannot be based on future information, such as the remaining processing time of unfinished jobs), nonpreemptive (processing of a job, once started, proceeds uninterruptedly to completion), and nonidling (the machine cannot stop working while there are jobs available) policies, denoted by $\mathcal{U}$, that minimizes the total expected (discounted or undiscounted) holding cost incurred to process all jobs. Note that the classical Smith's model corresponds to the special linear undiscounted case where $C_{\left(i, m_{i}\right)}=C_{i} m_{i}$.

Here we shall assume instead that holding cost rates are general separable, nondecreasing and convex functions on the number of jobs in each class, as stated next. Let us write the corresponding marginal costs rates as

$$
\Delta C_{\left(i, m_{i}\right)}=C_{\left(i, m_{i}\right)}-C_{\left(i, m_{i}-1\right)}, \quad m_{i} \geq 1, i \in N
$$

Assumption 1 The holding cost rates for each job class $i \in N$ are nondecreasing and convex: for $m_{i} \geq 1$,

(i) $\Delta C_{\left(i, m_{i}\right)} \geq 0$;

(ii) $\Delta C_{\left(i, m_{i}\right)} \geq \Delta C_{\left(i, m_{i}-1\right)}$.

In order to formulate the problem we associate to each scheduling policy $u \in \mathcal{U}$, job class $i \in N$, number of class $i$ jobs in system $1 \leq m_{i} \leq m_{i}^{0}$, and discount factor $\alpha \geq 0$, a performance measure $x_{\left(i, m_{i}\right)}^{u, \alpha}$, defined as the total expected $\alpha$-discounted time that there are 
$m_{i}$ class $i$ jobs in system under policy $u$. Letting $L_{i}(t)$ denote the number of class $i$ jobs in system at time $t$, we can express $x_{\left(i, m_{i}\right)}^{\pi, \alpha}$ as

$$
x_{\left(i, m_{i}\right)}^{u, \alpha}=E_{u}\left[\int_{0}^{\infty} 1\left\{L_{i}(t)=m_{i}\right\} e^{-\alpha t} d t\right], \quad\left(i, m_{i}\right) \in \widehat{N},
$$

where $\widehat{N}$ denotes the set of extended job classes defined by

$$
\widehat{N}=\left\{\left(i, m_{i}\right): i \in N, 1 \leq m_{i} \leq m_{i}^{0}\right\}
$$

The scheduling problem of concern is thus formulated as

$$
Z^{\mathrm{OPT}, \alpha}=\min _{u \in \mathcal{U}} \sum_{\left(i, m_{i}\right) \in \widehat{N}} C_{\left(i, m_{i}\right)} x_{\left(i, m_{i}\right)}^{u, \alpha}
$$

Note in (3) that the introduction of performance measures $x_{\left(i, m_{i}\right)}^{u, \alpha}$ allows us to incorporate the given nonlinear holding costs $C_{\left(i, m_{i}\right)}$ into a linear performance objective. This fact will play a central role in our solution approach, based on constructing and solving a linear programming (LP) formulation of problem (3).

\section{LP formulation and optimal index rule}

We next state our two main results in the paper.

(1) LP formulation. We present an LP formulation for problem (3), as follows. Let us define a family of extended class subsets $\mathcal{F} \subset 2^{\widehat{N}}$ by

$$
\mathcal{F}=\left\{S=\cup_{i \in N} S_{i}: S_{i} \in \mathcal{F}_{i}, i \in N\right\},
$$

where

$$
\mathcal{F}_{i}=\{\emptyset\} \cup\left\{\left\{\left(i, k_{i}\right), \ldots,\left(i, m_{i}^{0}\right)\right\}, 1 \leq k_{i} \leq m_{i}^{0}\right\}, \quad i \in N
$$

The LP formulation takes the form

$$
\begin{aligned}
Z^{\mathrm{LP}, \alpha}= & \min \sum_{\left(i, m_{i}\right) \in \widehat{N}} C_{\left(i, m_{i}\right)} x_{\left(i, m_{i}\right)} \\
& \text { subject to } \\
& \sum_{\left(i, m_{i}\right) \in S} A_{\left(i, m_{i}\right)}^{S, \alpha} x_{\left(i, m_{i}\right)} \geq b_{\alpha}(S), \quad S \in \mathcal{F} \backslash\{\widehat{N}\} \\
& \sum_{\left(i, m_{i}\right) \in \widehat{N}} A_{\left(i, m_{i}\right)}^{\widehat{N}, \alpha} x_{\left(i, m_{i}\right)}=b_{\alpha}(\widehat{N}) \\
& x_{\left(i, m_{i}\right)} \geq 0, \quad\left(i, m_{i}\right) \in \widehat{N}
\end{aligned}
$$


where the $A_{\left(i, m_{i}\right)}^{S, \alpha}$ 's (resp. $b_{\alpha}(S)$ 's) are positive (resp. nonnegative) coefficients. Problem (6) is a special type of the LPs over certain greedoid (cf. [9]) polyhedra we introduced and investigated in $[11,12]$. For arbitrary cost coefficients $C_{\left(i, m_{i}\right)},(6)$ is only an LP relaxation of scheduling problem (3): we shall show that the performance measures $x_{\left(i, m_{i}\right)}^{u, \alpha}$ achieved by admissible policies $u$ satisfy the constraints of LP (6), so that $Z^{\mathrm{LP}, \alpha} \leq Z^{\mathrm{OPT}, \alpha}$. We shall further establish that, under the admissible cost coefficients that satisfy Assumption 1, (6) is an exact LP formulation of (3), in that $Z^{\mathrm{LP}, \alpha}=Z^{\mathrm{OPT}, \alpha}$. Exploiting this fact leads to our next result.

(2) Optimal dynamic index rule. We define for each extended job class $\left(i, m_{i}\right) \in \widehat{N}$ an index $\gamma_{\left(i, m_{i}\right)}^{\alpha}$, and establish that it is optimal for scheduling problem (3) to sequence the jobs by giving higher priority to jobs whose current extended class has larger index. In the undiscounted case $\alpha=0$ the index is

$$
\gamma_{\left(i, m_{i}\right)}^{0}=\frac{\Delta C_{\left(i, m_{i}\right)}}{p_{i}}
$$

Note that in the classical case where $C_{\left(i, m_{i}\right)}=C_{i} m_{i}$ the above index reduces to Smith's: $\gamma_{\left(i, m_{i}\right)}^{0}=C_{i} / p_{i}$. In the discounted case, the index is

$$
\gamma_{\left(i, m_{i}\right)}^{\alpha}=\frac{E\left[e^{-\alpha \xi_{i}}\right] \Delta C_{\left(i, m_{i}\right)}}{E\left[\int_{0}^{\xi_{i}} e^{-\alpha t} d t\right]}=\frac{\alpha \phi_{i}(\alpha) \Delta C_{\left(i, m_{i}\right)}}{1-\phi_{i}(\alpha)}, \quad\left(i, m_{i}\right) \in \widehat{N} .
$$

It is clear from the above expressions that the index $\gamma_{\left(i, m_{i}\right)}^{\alpha}$ has the following economic interpretation: index $\gamma_{\left(i, m_{i}\right)}^{\alpha}$ is the marginal expected (discounted) holding cost rate reduction per unit of expected (discounted) processing time corresponding to processing a class $i$ job when there are $m_{i}$ such jobs in system.

Note further that the resulting dynamic index policy has a dynamic threshold structure: if the current index $\gamma_{\left(i, m_{i}\right)}^{\alpha}$ is the larger, the machine should continue processing class $i$ jobs until their number in system falls below a certain threshold level (determined by all current indices), at which time processing switches to another class.

\section{Solution approach via partial conservation laws: background and outline}

Our approach to the solution of problem (3) by an index rule is based on obtaining an $L P$ relaxation with strong structural properties, which is exact under the conditions on holding costs given in Assumption 1. Such LP relaxation results from the satisfaction by performance measures $x_{\left(i, m_{i}\right)}^{u, \alpha}$ of a family of partial conservation laws (PCL). To better grasp and put the PCL in context, we next briefly survey the use of conservation laws in the LP formulation and solution by index rules of scheduling problems.

In short, the classical results mentioned above on the optimality of index rules for single machine scheduling have later been shown to result from the strong structural properties of the underlying system's achievable performance region, (i.e., the convex hull of performance 
vectors achievable under admissible policies $u \in \mathcal{U}$; cf. $[1,3])$ : this is a polytope with special structure (an (extended) polymatroid), whose constraints formulate work conservation laws, and whose vertices are achieved by priority-index rules.

To illustrate, the simplest example is again Smith's [18] classical model. Associate with each class $i$ and scheduling policy $u$ a performance measure $x_{i}^{u}$, representing the sum of completion times of class $i$ jobs under that policy. Then, as established by Queyranne in [13], the achievable region corresponding to performance vector $\mathbf{x}^{u}=\left(x_{i}^{u}\right)_{i \in N}$ is a polytope in $\mathbb{R}_{+}^{n}$ of the form

$$
\begin{aligned}
& \sum_{i \in S} p_{i} x_{i} \geq b(S), \quad S \in 2^{N} \backslash\{N\} \\
& \sum_{i \in N} p_{i} x_{i}=b(N)
\end{aligned}
$$

The constraints (7) and (8) formulate simple work conservation relations. Equality is achieved in (7) under any policy that gives higher priority to classes in $S$. The resulting (scaled) polytope is a polymatroid: the well-known optimality of the greedy algorithm for LP over polymatroids (cf. [4]) thus explains the optimality of Smith's index rule for the scheduling problem.

A more comprehensive framework, based on the concept of generalized conservation laws (GCL), was introduced in [1] and shown to provide a unifying account of the optimality of index rules in the classical scheduling models mentioned above [18, 16, 2, 8, 7, 19, 5, 6, 10, 22]: under GCL, the achievable region is a polytope in $\mathbb{R}_{+}^{n}$ described by constraints of the form

$$
\begin{aligned}
\sum_{i \in S} A_{i}^{S} x_{i} & \geq b(S), \quad S \in 2^{N} \backslash\{N\} \\
\sum_{i \in N} A_{i}^{N} x_{i} & =b(N)
\end{aligned}
$$

where $A_{i}^{S}>0$ for all $i \in N, S \in 2^{N}$, which define an extended polymatroid. Equality is achieved in (9) by any policy that gives priority to classes in $S$. An LP over such polyhedra is solved in $n$ steps by Klimov's [8] adaptive-greedy algorithm, which computes a set of optimal priority indices for a linear performance objective $\left(\min \sum_{i} C_{i} x_{i}\right)$.

For certain models, however, such as the one of concern in this paper (cf. (3)), establishing the optimality of index rules under arbitrary linear objectives (complete indexability) appears as too strong a requirement. The relevant concern is instead to establish the optimality of a family of index rules with special structure under a range of admissible linear objectives (partial indexability). The concept of PCL, introduced in [11] and further developed in [12], is an extension of GCL that provides a framework for establishing such partial indexability property. In short, PCL are obtained by relaxing the requirement in the GCL that inequality (9) holds for all class subsets $S \in 2^{N} \backslash\{N\}$, and requiring instead that it holds for $S \in \mathcal{F} \backslash\{N\}$, where $\mathcal{F} \subseteq 2^{N}$ is an appropriate family of feasible class subsets that may receive higher priority. The resulting polytope is a form of greedoid polyhedron with strong 
structural and algorithmic properties, which we introduced and investigated extensively in $[11,12]$. We called in [11] $P C L$-indexable the class of scheduling problems which are solved optimally by priority index policies due to the satisfaction of PCL.

\section{Structure of the paper}

The rest of the paper is structured as follows. In section 2 we review the PCL framework, in a form adapted to the model under study. In section 3 we establish that the system

performance measures $x_{\left(i, m_{i}\right)}^{u, \alpha}$ satisfy PCL corresponding to the family of feasible extended class subsets $\mathcal{F}$ defined in (4). In section 4 we characterize the range of admissible costs under which the scheduling problem is PCL-indexable as those costs satisfying Assumption 1 , and derive the resulting optimal priority indices $\gamma_{\left(i, m_{i}\right)}^{\alpha}$.

\section{Partial conservation laws}

In this section we review the PCL framework outlined above, which we introduced in [11] and further developed in [12]. Consider a general dynamic and (optionally) stochastic service system catering to a finite set $N$ of $n<\infty$ job classes. Service resources (e.g., servers) are to be allocated over time to jobs vying for their attention, on the basis of a scheduling policy $u$, which belongs in a space $\mathcal{U}$ of admissible policies. The performance of policy $u \in \mathcal{U}$ over job class $i \in N$ is given by performance measure $x_{i}^{u} \geq 0$, which we assume to be an expectation. We denote by $\mathbf{x}^{u}=\left(x_{i}^{u}\right)_{i \in N}$ the corresponding performance vector. We further assume the system admits a consistent notion of service priority among classes: to each full ordered string $\boldsymbol{\pi}=\left(\pi_{1}, \ldots, \pi_{n}\right)$ of $N$, which spans all the classes, is associated a corresponding $\boldsymbol{\pi}$-priority policy, which assigns higher priority to class $\pi_{i}$ over class $\pi_{j}$ if $i<j$, so that class $\pi_{1}$ has top priority. We refer to all such policies as priority policies. We further say that a policy gives priority to classes in a subset $S \subseteq N(S$-jobs $)$ if it gives priority to each class $i \in S$ over each class $j \in S^{c}=N \backslash S$.

Consider now, for a given cost vector $\mathbf{C}=\left(C_{i}\right)_{i \in N} \in \mathbb{R}^{n}$, the optimal scheduling problem

$$
Z^{\mathrm{OPT}}(\mathbf{C})=\min \left\{\sum_{i \in N} C_{i} x_{i}^{u}: u \in \mathcal{U}\right\},
$$

which involves finding an admissible scheduling policy that minimizes the linear performance objective in (11), and computing the corresponding optimum value $Z^{\mathrm{OPT}}(\mathbf{C})$.

A wide variety of scheduling problems fitting formulation (11) possess the following structural property, which we call complete indexability: to each cost vector $\mathbf{C} \in \mathbb{R}^{n}$ there corresponds an index vector $\gamma(\mathbf{C})=\left(\gamma_{i}(\mathbf{C})\right)_{i \in \mathcal{N}}$ such that the corresponding priority-index policy (which gives higher priority to classes with larger indices) is optimal for problem (11). A general framework providing a sufficient condition for problem (11) to be completely indexable was presented in [1]: satisfaction by performance vector $\mathbf{x}^{u}$ of generalized conservation 
laws (GCL) implies complete indexability; furthermore, under GCL the optimal indices can be efficiently computed through an $n$-step adaptive-greedy algorithm due to Klimov [8]. In [11] we called $P C L$-indexable the scheduling problems that satisfy PCL.

In other scheduling models, such as the one of concern in this paper, complete indexability appears as too strong a requirement: the relevant concern is instead to establish the optimality of a given family of priority policies with special structure under a limited range of admissible linear performance objectives. If such is the case, then there exists an optimal vector of priority indices $\gamma(\mathbf{C})$, yet it is defined only over a domain $\mathcal{D} \subseteq \mathbb{R}^{n}$ of admissible costs $\mathbf{C}$, which further yields priorities having the required structure. We call such property partial indexability.

We review next the general framework for establishing partial indexability we introduced in [11], based on the satisfaction by performance measures of a relaxed version of the GCL, which we call partial conservation laws (PCL).

We shall represent a given family of priority policies with special structure by a set system $(N, \mathcal{F})$ defined over the ground set $N$ of classes, so that $\mathcal{F} \subseteq 2^{N}$ is the family of feasible class subsets that may be assigned higher priority under the given policy family.

Definition $1\left(\mathcal{F}\right.$-policies) We say that a priority policy $\boldsymbol{\pi}=\left(\pi_{1}, \ldots, \pi_{n}\right)$ is an $\mathcal{F}$-policy, and write $\boldsymbol{\pi} \in \mathcal{F}$, if

$$
\left\{\pi_{1}, \ldots, \pi_{k}\right\} \in \mathcal{F}, \quad 1 \leq k \leq n
$$

Note that under Assumption 1 set system $(N, \mathcal{F})$ is a special type of greedoid (cf. [9]). It will be convenient to consider further the complementary family $\mathcal{F}^{c}=\left\{S^{c}: S \in \mathcal{F}\right\}$ of job class subsets that may receive lower priority, where we write $S^{c}=N \backslash S$. We impose on set system $(N, \mathcal{F})$ the requirements stated next, which are motivated by algorithmic considerations.

Assumption 2 Set system $(N, \mathcal{F})$ satisfies the following conditions:

(i) Accessibility: if $\emptyset \neq S \in \mathcal{F}$ then there exists $i \in S$ such that $S \backslash\{i\} \in \mathcal{F}$;

(ii) Augmentability: if $N \neq S \in \mathcal{F}$ then there exists $i \in S^{c}$ such that $S \cup\{i\} \in \mathcal{F}$.

Note that under Assumption 2 set system $(N, \mathcal{F})$ is a greedoid (cf. [9]). Furthermore, if $(N, \mathcal{F})$ satisfies Assumption 2 then so does its complementary set system $\left(N, \mathcal{F}^{c}\right)$, and it follows that $\emptyset$ and $N$ are members of both $\mathcal{F}$ and $\mathcal{F}^{c}$.

Definition 1 (Partial conservation laws (PCL)) Performance vector $\mathbf{x}(u)$ satisfies partial conservation laws with respect to the family of $\mathcal{F}$-policies if there exist coefficients $A_{i}^{S}>0$ for $i \in S$ and $S \in \mathcal{F}$ such that, letting

$$
b(S)=\inf \left\{\sum_{i \in S} A_{i}^{S} x_{i}(u): u \in \mathcal{U}\right\}, \quad S \in \mathcal{F},
$$


the following identities hold: for $S \in \mathcal{F} \backslash\{N\}$,

$$
\sum_{i \in S} A_{i}^{S} x_{i}^{u}=b(S), \quad \text { under any } \mathcal{F} \text {-policy } u \text { giving priority to } S \text {-jobs; }
$$

and, for $S=N$,

$$
\sum_{i \in N} A_{i}^{N} x_{i}^{u}=b(N), \quad \text { under any policy } u \in \mathcal{U}
$$

Note that the GCL in [1] correspond to the special case of the PCL above where $\mathcal{F}=2^{N}$. For a scheduling problem that satisfies the PCL in Definition 1, consider the LP problem

$$
\begin{aligned}
Z^{\mathrm{LP}}(\mathbf{C})= & \min \sum_{i \in N} C_{i} x_{i} \\
& \text { subject to } \\
y^{S}: & \sum_{i \in S} A_{i}^{S} x_{i} \geq b(S), \quad S \in \mathcal{F} \backslash\{N\} \\
y^{N}: & \sum_{i \in N} A_{i}^{N} x_{i}=b(N) \\
& x_{i} \geq 0, \quad i \in N,
\end{aligned}
$$

where we have attached to each constraint a corresponding dual variable $y^{S}$. For each cost vector $\mathbf{C} \in \mathbb{R}^{n}$, the PCL imply that problem (14) is an LP relaxation of scheduling problem (11), so that $Z^{\mathrm{LP}}(\mathbf{C}) \leq Z^{\mathrm{OPT}}(\mathbf{C})$. We shall identify next a range of cost vectors $\mathbf{C}$ for which (14) is an exact LP formulation of problem (11), in that $Z^{\mathrm{LP}}(\mathbf{C})=Z^{\mathrm{OPT}}(\mathbf{C})$.

The region $\mathcal{D}(\mathcal{F}) \subseteq \mathbb{R}^{n}$ of $\mathcal{F}$-admissible costs is defined in terms of the adaptive-greedy algorithm $\operatorname{AG}(\cdot \mid \mathcal{F})$ described in Figure 1. This algorithm takes as input a cost vector $\mathbf{C}$, and produces as output a triple: a boolean variable $A D M I S S I B L E \in\{Y E S, N O\}$; an index vector $\boldsymbol{\gamma}=\left(\gamma_{i}\right)_{i \in N}$; and a full ordered string $\boldsymbol{\pi}=\left(\pi_{1}, \ldots, \pi_{n}\right)$ of $N$. We thus define the $\mathcal{F}$ admissible cost region $\mathcal{D}(\mathcal{F})$ as the domain of algorithm $\operatorname{AG}(\cdot \mid \mathcal{F})$. That is, as the set of input cost vectors $\mathbf{C}$ for which the algorithm returns an output having ADMISSIBLE $=Y E S$. The admissibility test carried out by the algorithm is

$$
\gamma_{\pi_{1}} \geq \ldots \geq \gamma_{\pi_{n}}
$$

Note that in the special GCL case $\mathcal{D}\left(2^{N}\right)=\mathbb{R}^{n}$, and hence all costs are then admissible.

The next definition draws on the notion of PCL to define a class of scheduling problems that, as stated in Theorem 1 below, are partially indexable.

Definition 2 (PCL/GCL-indexability) Scheduling problem (11) is said to be $P C L$-indexable with respect to the family of $\mathcal{F}$-policies if

(i) it satisfies PCL with respect to $\mathcal{F}$-policies; and

(ii) cost vector $\mathbf{C}$ is $\mathcal{F}$-admissible: $\mathbf{C} \in \mathcal{D}(\mathcal{F})$.

If (i) holds with $\mathcal{F}=2^{N}$ we say the problem is $G C L$-indexable. 


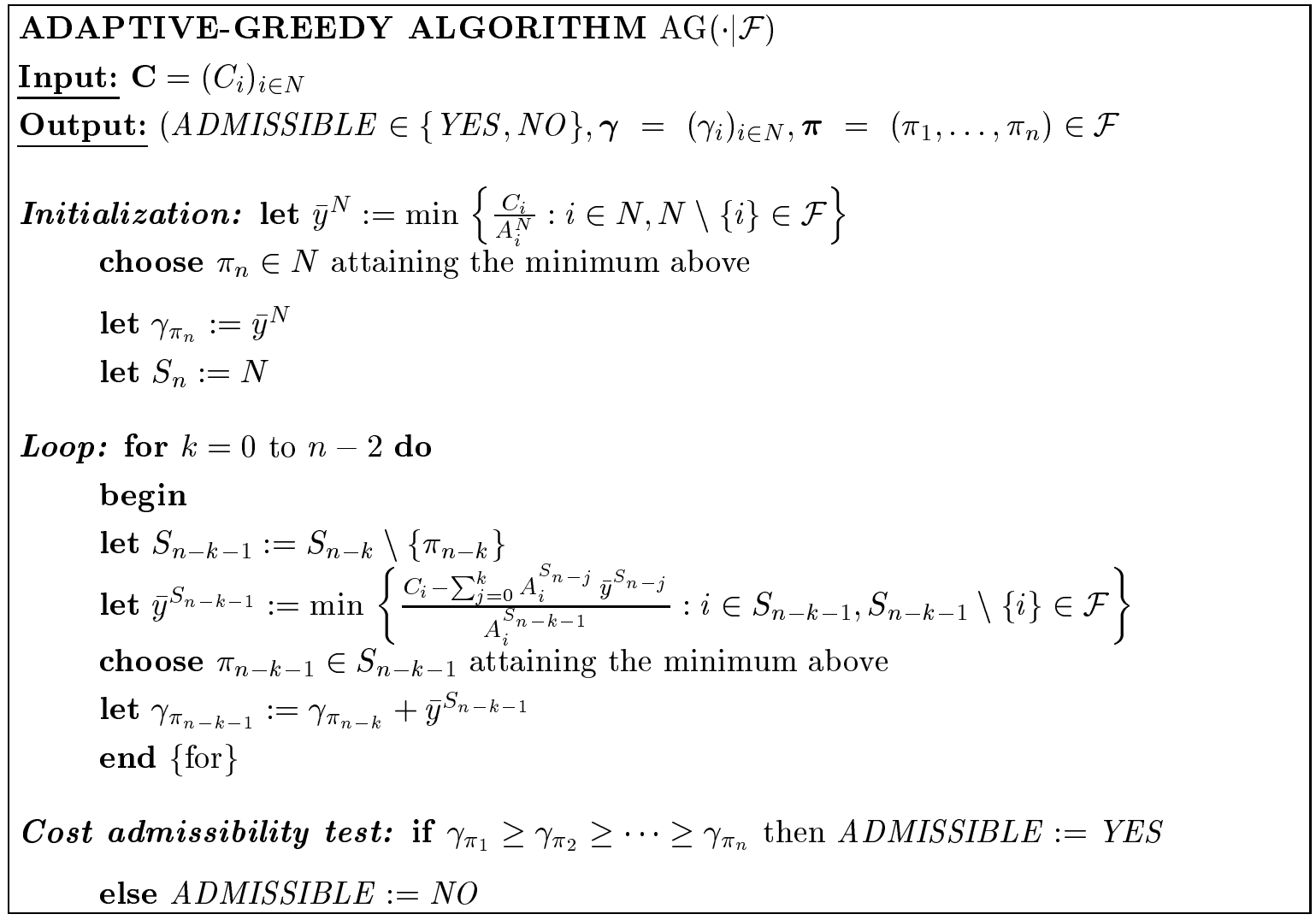

Figure 1: Adaptive-greedy algorithm $\operatorname{AG}(\cdot \mid \mathcal{F})$. 
The next result shows that PCL-indexable problems are indeed indexable. Let the output of adaptive-greedy algorithm $\operatorname{AG}(\cdot \mid \mathcal{F})$ corresponding to input $\mathbf{C}$ be given by the triple $(A D M I S S I B L E, \boldsymbol{\gamma}, \boldsymbol{\pi})$.

Theorem 1 (Indexability under PCL) Assume problem (11) is PCL-indexable with respect to the family of $\mathcal{F}$-policies. Then, the problem is solved optimally by any $\mathcal{F}$-policy that gives higher priority to class $i$ over class $j$ if $\gamma_{i} \geq \gamma_{j}$, for $i, j \in N$.

Note that in the special case where $\mathcal{F}=2^{N}$, algorithm $\operatorname{AG}(\cdot \mid \mathcal{F})$ reduces to the well-known adaptive-greedy algorithm due to Klimov [8], and Theorem 1 then yields the optimality of priority-index policies under arbitrary linear objectives, as established in the GCL framework (cf. [1]).

The proof of Theorem 1 is provided in $[11,12]$, but we review here its main point. We showed in those papers that the above output of the adaptive-greedy algorithm satisfies the following properties:

(1) Objective decomposition: The performance objective decomposes as follows. Let $S_{k}=\left\{\pi_{n-k+1}, \ldots, \pi_{n}\right\}$, for $1 \leq k \leq n$.

$$
\sum_{i \in N} C_{i} x_{i}^{u}=\gamma_{\pi_{n}} \sum_{i \in N} A_{i}^{N} x_{i}^{u}+\left(\gamma_{\pi_{n-k}}-\gamma_{\pi_{n-k+1}}\right) \sum_{k=1}^{n-1} \sum_{i \in S_{n-k}} A_{i}^{S_{n-k}} x_{i}^{u} .
$$

(2) Optimal dual solution: Under cost admissibility condition (15), an optimal dual solution to LP problem (14) is obtained by setting $y^{N}=\gamma_{\pi_{n}}, y^{S_{k}}=\gamma_{\pi_{n-k}}-\gamma_{\pi_{n-k+1}}$, for $1 \leq k \leq n-1$. See Figure 1 .

(3) Optimal priority policy: Under cost admissibility condition (15), satisfaction of the PCL in Definition 1, combined with identity (16), yields directly the the result in Theorem 1.

Figure 2 illustrates the inclusion relations between different types of scheduling problems that results from the introduction of the classes of PCL-indexable and GCL-indexable problems.

\subsection{Project scheduling and index decomposition}

It was shown in [1] that in the GCL framework $\left(\mathcal{F}=2^{N}\right.$ above) a stronger index decomposition property holds under appropriate conditions. That property explains that in some scheduling models with multiple projects, such as classical multiarmed bandits, the priority indices are defined in terms of individual projects. An analogous index decomposition property holds in the PCL framework, as we showed in $[11,12]$, and review next. Consider the special case of the general model above which corresponds to the problem of scheduling a collection of $m$ projects, where project $k$ has state space $N_{k}$, for $1 \leq k \leq m$. Assume the project state 


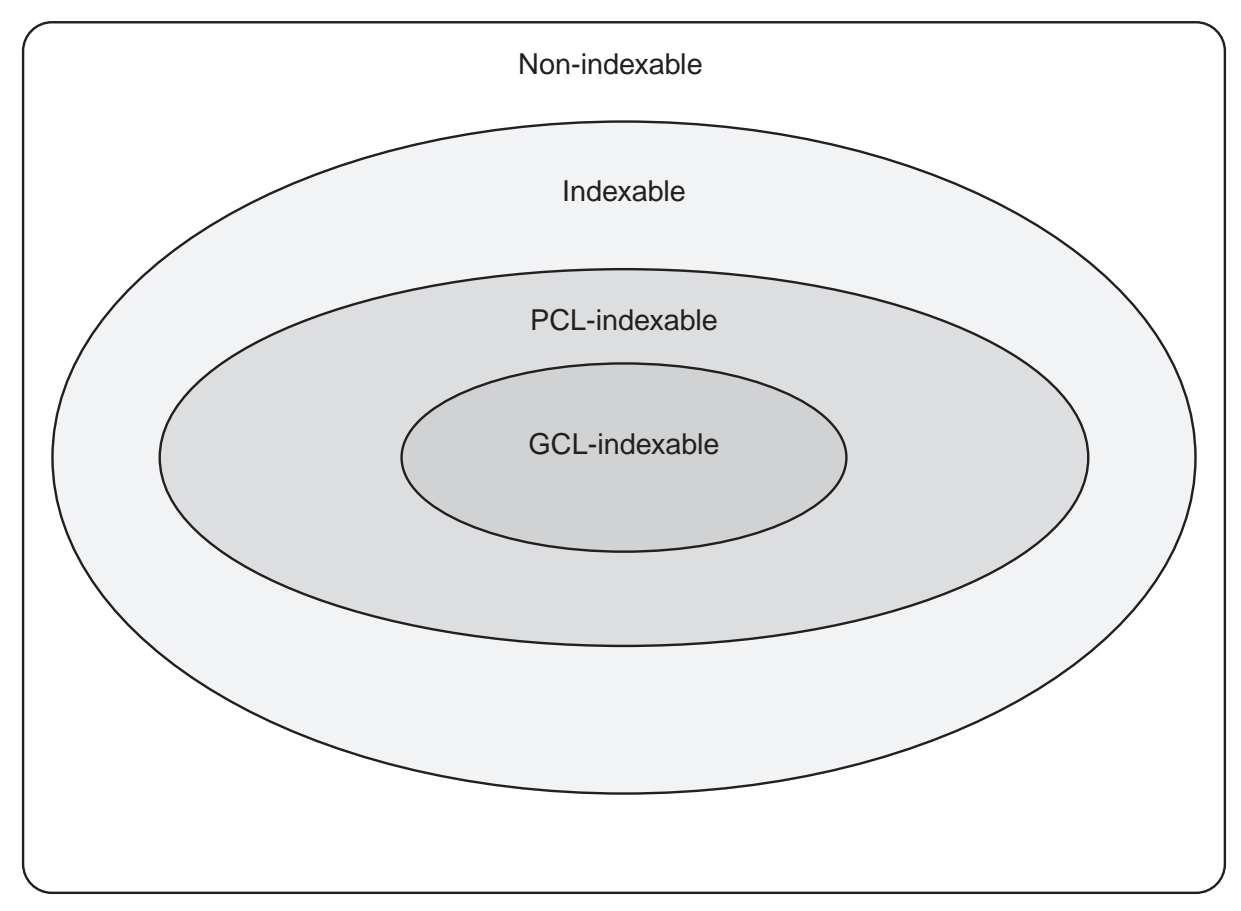

Figure 2: Classification of scheduling problems.

spaces are disjoint, and consider the aggregate state space $N=\cup_{k=1}^{m} N_{k}$. Note that in this setting, project states play the role of job classes in the general framework above. Suppose it can be established that a performance vector $\mathbf{x}^{u}=\left(x_{i}^{u}\right)_{i \in N}$ for the system satisfies PCL with respect to the family of $\mathcal{F}$-policies, with parameters $A_{i}^{S}$. Let

$$
\mathcal{F}_{k}=\left\{S \in \mathcal{F}: S \subseteq N_{k}\right\} \quad \text { and } \quad \mathcal{F}_{k}^{c}=\left\{S \in 2^{N_{k}}: N_{k} \backslash S \in \mathcal{F}_{k}\right\}, \quad 1 \leq k \leq m,
$$

and suppose the conditions given next hold.

Assumption 3 For $1 \leq k \leq m$,

(i) $\mathcal{F}_{k}=\left\{S \cap N_{k}: S \in \mathcal{F}\right\}$;

(ii) set system $\left(N_{k}, \mathcal{F}_{k}\right)$ satisfies Assumption 2;

(iii) $A_{i}^{S \cap N_{k}}=A_{i}^{S}, \quad i \in S \cap N_{k}, S \in \mathcal{F}$.

Let $\left(A D M I S S I B L E_{k}, \boldsymbol{\gamma}^{k}, \boldsymbol{\pi}^{k}\right)$ be the output of algorithm $\mathrm{AG}\left(\cdot \mid \mathcal{F}_{k}\right)$ on input $\mathbf{C}^{k}=\left(C_{i}\right)_{i \in N_{k}}$, for $1 \leq k \leq m$, and let $(A D M I S S I B L E, \boldsymbol{\gamma}, \boldsymbol{\pi})$ be the output of algorithm $\mathrm{AG}(\cdot \mid \mathcal{F})$ on input $\mathbf{C}=\left(C_{i}\right)_{i \in N}$.

Theorem 2 (Index decomposition) Under Assumption (3),

(a) $A D M I S S I B L E=Y E S$ if and only if $A D M I S S I B L E_{k}=Y E S$ for $1 \leq k \leq m$.

If ADMISSIBLE $=Y E S$, then

(b) $\gamma_{i}=\gamma_{i}^{k}$, for $1 \leq k \leq m$; and

(c) it is optimal to give higher priority to projects with larger indices. 


\section{Derivation of PCL}

In this section we establish that system performance measures $x_{\left(i, m_{i}\right)}^{u, \alpha}($ cf. (1)) satisfy PCL with respect to the family of feasible extended class subsets $\mathcal{F} \subset 2^{\widehat{N}}$ defined in (4), where $\widehat{N}$ is the set of extended job classes $\left(i, m_{i}\right)$ (cf. (2)).

We start by introducing the random variables given next, which describe the system evolution. Let $S=\cup_{i \in N} S_{i} \in \mathcal{F}$, with the nonempty $S_{i}$ 's being given by

$$
S_{i}=\left\{\left(i, k_{i}\right), \ldots,\left(i, m_{i}^{0}\right)\right\}
$$

$t_{\left(i, m_{i}\right)}=$ time at which processing of the $m_{i}$ th class $i$ job (starting from the 1 st in line) starts;

$\xi_{\left(i, m_{i}\right)}=$ processing time of the $m_{i}$ th class $i$ job;

$W(t)=$ system workload (total remaining processing time) at time $t$;

$W_{\left(i, m_{i}\right)}(t)=$ workload component at time $t$ due to the $m_{i}$ th class $i$ job;

$W_{S}(t)=$ workload due to class $i$ jobs in positions $k_{i}$ up to $m_{i}^{0}(S$-workload $)$ at time $t$; that

is, $W_{S}(t)=\sum_{\left(i, m_{i}\right) \in S} W_{\left(i, m_{i}\right)}(t)$. Note $W_{\widehat{N}}(t)=W(t)$.

$T=$ finishing time of all jobs (or, equivalently, sum of the processing time of all jobs).

$T_{S}=W_{S}(0)$. Note $T_{\widehat{N}}=T$.

The nonidling assumption on admissible scheduling policies $u \in \mathcal{U}$ clearly implies that $T$ is policy-invariant, having expected value

$$
E[T]=\sum_{i \in N} m_{i}^{0} p_{i}
$$

Furthermore, by independence of the job processing times, its variance is given by

$$
\operatorname{Var}[T]=\sum_{i \in N} m_{i}^{0} \sigma_{i}^{2}
$$

and its Laplace transform $\phi_{T}(\alpha)$ is

$$
\phi_{T}(\alpha)=E\left[e^{-\alpha T}\right]=\prod_{i \in N} \phi_{i}^{m_{i}^{0}}(\alpha) .
$$

Similarly, for each feasible expanded class set $S=\cup_{i \in N} S_{i} \in \mathcal{F}$, with each $S_{i} \in \mathcal{F}_{i}$ (see (5)) we have the following. Under policies $u \in \mathcal{U}$ that gives higher priority to expanded classes in $S, T_{S}$ is invariant, having expected value $\left(\left|S_{i}\right|\right.$ denotes the cardinality of set $\left.S_{i}\right)$

$$
E\left[T_{S}\right]=\sum_{i \in N}\left|S_{i}\right| p_{i}
$$


variance

$$
\operatorname{Var}\left[T_{S}\right]=\sum_{i \in N}\left|S_{i}\right| \sigma_{i}^{2}
$$

and Laplace transform

$$
\phi_{T_{S}}(\alpha)=E\left[e^{-\alpha T_{S}}\right]=\prod_{i \in N} \phi_{i}^{\left|S_{i}\right|}(\alpha) .
$$

Note further that, for $1 \leq m_{i} \leq m_{i}^{0}-1$,

$$
\begin{aligned}
& x_{\left(i, m_{i}\right)}^{u, \alpha}=E_{u}\left[\int_{t_{\left(i, m_{i}+1\right)}+\xi_{\left(i, m_{i}+1\right)}}^{t_{\left(i, m_{i}\right)}+\xi_{\left(i, m_{i}\right)}} e^{-\alpha t} d t\right] \\
& =\left\{\begin{array}{cc}
\frac{1}{\alpha} \phi_{i}(\alpha)\left(E_{u}\left[e^{-\alpha t_{\left(i, m_{i}+1\right)}}\right]-E_{u}\left[e^{\left.\left.-\alpha t_{\left(i, m_{i}\right)}\right]\right)}\right.\right. & \text { if } \alpha>0 ; \\
E_{u}\left[t_{\left(i, m_{i}\right)}\right]-E_{u}\left[t_{\left(i, m_{i}+1\right)}\right] & \text { if } \alpha=0 .
\end{array}\right.
\end{aligned}
$$

Furthermore,

$$
x_{\left(i, m_{i}^{0}\right)}^{u, \alpha}=E_{u}\left[\int_{0}^{t_{\left(i, m_{i}^{0}\right)}+\xi_{\left(i, m_{i}^{0}\right)}} e^{-\alpha t} d t\right]=\left\{\begin{array}{cl}
\frac{1}{\alpha}-\frac{1}{\alpha} \phi_{i}(\alpha) E_{u}\left[e^{\left.-\alpha t_{\left(i, m_{i}^{0}\right)}\right]}\right. & \text { if } \alpha>0 \\
E_{u}\left[t_{\left(i, m_{i}^{0}\right)}\right]+p_{i} & \text { if } \alpha=0 .
\end{array}\right.
$$

It follows that

$$
\sum_{k_{i}=m_{i}}^{m_{i}^{0}} x_{\left(i, k_{i}\right)}^{u, \alpha}=\left\{\begin{array}{cc}
\frac{1}{\alpha}-\frac{1}{\alpha} \phi_{i}(\alpha) E_{u}\left[e^{\left.-\alpha t_{\left(i, m_{i}\right)}\right]}\right. & \text { if } \alpha>0 \\
E_{u}\left[t_{\left(i, m_{i}\right)}\right]+p_{i} & \text { if } \alpha=0 .
\end{array}\right.
$$

For each feasible set of the form $S=\cup_{i \in N} S_{i} \in \mathcal{F}$, with

$$
S_{i}=\left\{\left(i, k_{i}\right), \ldots,\left(i, m_{i}^{0}\right)\right\} \in \mathcal{F}_{i}, \quad i \in N
$$

let us write

$$
A_{\left(i, m_{i}\right)}^{S}=A_{\left(i, m_{i}\right)}^{S_{i}},
$$

where

$$
A_{\left(i, m_{i}\right)}^{S_{i}, \alpha}=\left\{\begin{array}{cc}
\left(m_{i}-k_{i}+1\right) \frac{1-\phi_{i}(\alpha)}{\alpha \phi_{i}(\alpha)} & \text { if } \alpha>0 \\
\left(m_{i}-k_{i}+1\right) p_{i} & \text { if } \alpha=0
\end{array}\right.
$$

Let us further define, for $\alpha>0$,

$$
b_{\alpha}(S)=\sum_{i \in N}\left|S_{i}\right| \frac{1}{\alpha^{2}} \frac{1-\phi_{i}(\alpha)}{\phi_{i}(\alpha)}-\frac{1}{\alpha^{2}}\left(1-\phi_{T_{S}}(\alpha)\right)
$$

and, for $\alpha=0$,

$$
b(S)=\frac{1}{2} \sum_{i \in N}\left|S_{i}\right| p_{i}^{2}+\frac{1}{2}\left(\sum_{i \in N}\left|S_{i}\right| p_{i}\right)^{2} .
$$

Note that

$$
b_{0}(\widehat{N})=\frac{1}{2} \sum_{i \in N} m_{i}^{0} p_{i}^{2}+\frac{1}{2}\left(\sum_{i \in N} m_{i}^{0} p_{i}\right)^{2}
$$




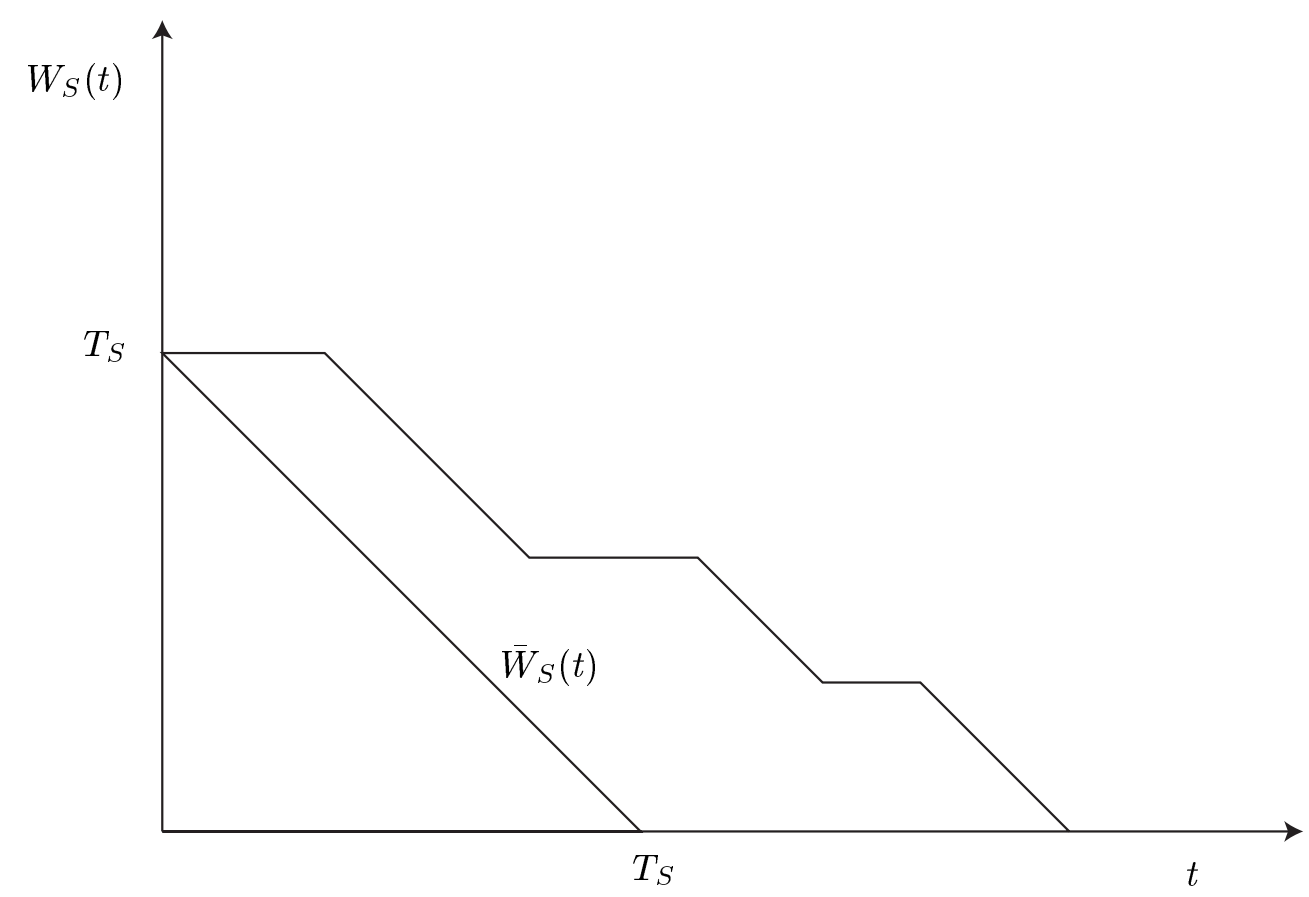

Figure 3: Sample path of workload component $W_{S}(t)$ and its lower bound $\bar{W}_{S}(t)$.

Theorem 3 (PCL) Performance measures $x_{\left(i, m_{i}\right)}^{u, \alpha}$ satisfy the following PCL with respect to the family of $\mathcal{F}$-policies: under any policy $u \in \mathcal{U}$,

(a) for each feasible expanded class set $S \in \mathcal{F}$,

$$
\sum_{\left(i, m_{i}\right) \in S} A_{\left(i, m_{i}\right)}^{S, \alpha} x_{\left(i, m_{i}\right)}^{u, \alpha} \geq b_{\alpha}(S)
$$

with equality in (20) if the policy gives higher priority to expanded classes in $S$; and (b)

$$
\sum_{\left(i, m_{i}\right) \in \hat{N}} A_{\left(i, m_{i}\right)}^{\hat{N}, \alpha} x_{\left(i, m_{i}\right)}^{u, \alpha}=b_{\alpha}(\hat{N})
$$

\section{Proof}

(a) We start by proving the equality part. Let $S=\cup_{i \in N} S_{i} \in \mathcal{F}$, with $S_{i} \in \mathcal{F}_{i}$. Write the nonempty $S_{i}$ 's as $S_{i}=\left\{\left(i, k_{i}\right), \ldots,\left(i, m_{i}^{0}\right)\right\}$. Let $u \in \mathcal{U}$ be an admissible scheduling policy, and consider the corresponding $S$-workload sample path $\left\{W_{S}(t), 0 \leq t \leq T\right\}$. Now, the nonidleness assumption implies that such $S$-workload sample path is invariant under policies $u$ that give higher priority to $S$-jobs. Denote such invariant $S$-workload by $\bar{W}_{S}(t)$. Furthermore, it is clear that, under any other admissible policy $u$ it holds that

$$
W_{S}(t) \geq \bar{W}_{S}(t), \quad 0 \leq t \leq T
$$

See Figure 3. 


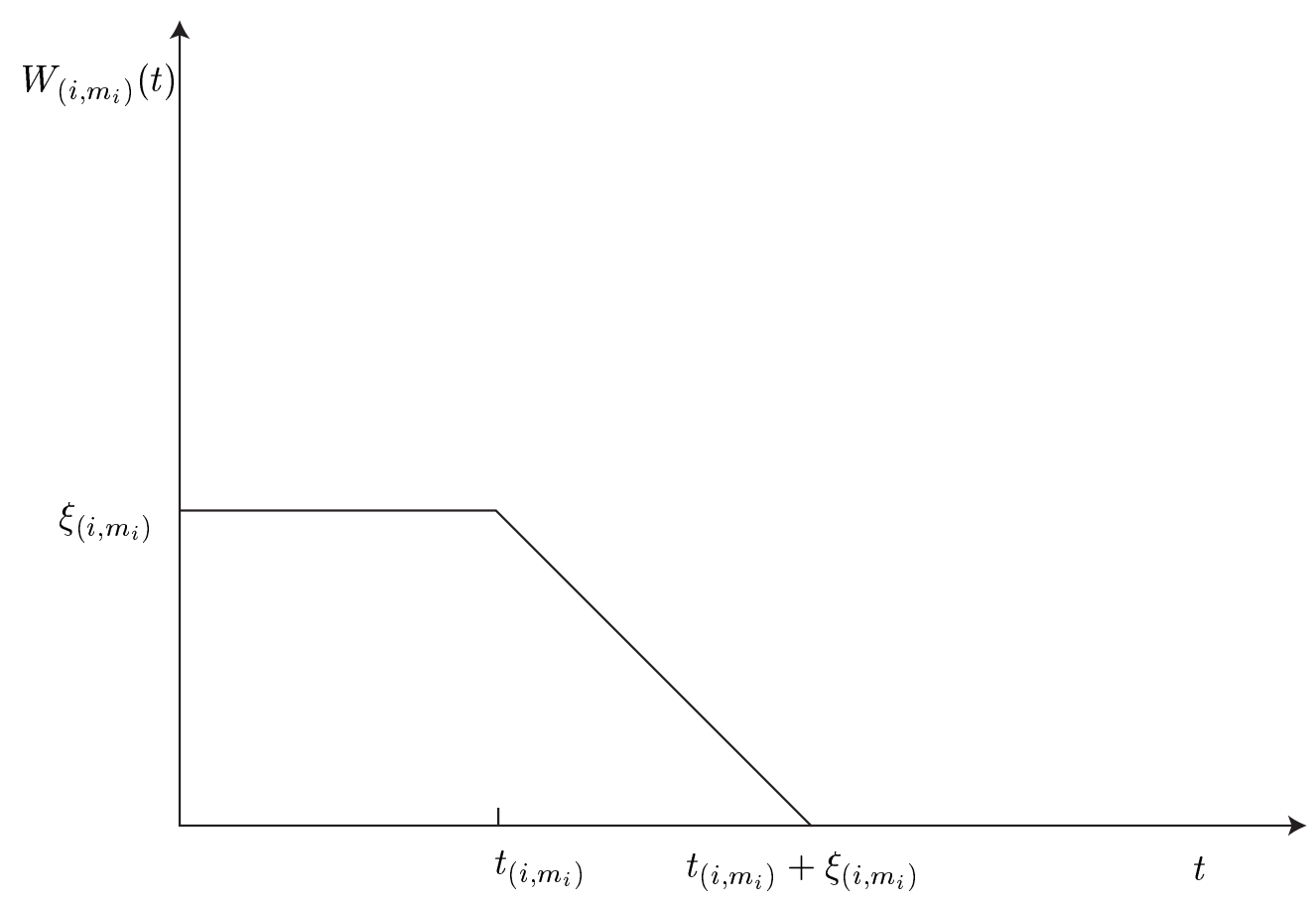

Figure 4: Sample path of workload component $W_{\left(i, m_{i}\right)}(t)$.

Therefore, the corresponding total discounted $S$-workload satisfies the inequality

$$
\begin{aligned}
E_{u}\left[\int_{0}^{T} W_{S}(t) e^{-\alpha t} d t\right] & \geq E_{u}\left[\int_{0}^{T} \bar{W}_{S}(t) e^{-\alpha t} d t\right] \\
& =E_{u}\left[\int_{0}^{T_{S}}\left(T_{S}-t\right) e^{-\alpha t} d t\right] \\
& =\left\{\begin{array}{cc}
\frac{1}{\alpha^{2}}\left(\alpha E\left[T_{S}\right]+E\left[e^{-\alpha T_{S}}\right]-1\right) & \text { if } \alpha>0 \\
\frac{1}{2} E\left[T_{S}^{2}\right] & \text { if } \alpha=0
\end{array}\right.
\end{aligned}
$$

with equality being achieved in (24) under any admissible policy that gives higher priority to $S$-jobs.

We now reformulate the left hand side of $(23)$ in terms of the $x_{\left(i, m_{i}\right)}^{u, \alpha}$ 's. It is easily seen (cf. Figure 4) that

$$
\begin{aligned}
E_{u}\left[\int_{0}^{T} W_{\left(i, m_{i}\right)}(t) e^{-\alpha t} d t\right] & =E_{u}\left[\int_{0}^{t_{\left(i, m_{i}\right)}} \xi_{\left(i, m_{i}\right)} e^{-\alpha t} d t+\int_{t_{\left(i, m_{i}\right)}}^{t_{\left(i, m_{i}\right)}+\xi_{\left(i, m_{i}\right)}}\left(t_{\left(i, m_{i}\right)}+\xi_{\left(i, m_{i}\right)}-t\right) e^{-\alpha t} d t\right] \\
& =\left\{\begin{array}{cl}
\frac{1}{\alpha} p_{i}-\frac{1}{\alpha^{2}}\left(1-\phi_{i}(\alpha)\right) E_{u}\left[e^{-\alpha t_{\left(i, m_{i}\right)}}\right] & \text { if } \alpha>0 \\
p_{i} E_{u}\left[t_{\left(i, m_{i}\right)}\right]+\frac{1}{2}\left(p_{i}^{2}+\sigma_{i}^{2}\right) & \text { if } \alpha=0 .
\end{array}\right.
\end{aligned}
$$


We can thus write

$$
\begin{aligned}
E_{u}\left[\int_{0}^{T} W_{S}(t) e^{-\alpha t} d t\right] & =\sum_{\left(i, m_{i}\right) \in S} E_{u}\left[\int_{0}^{T} W_{\left(i, m_{i}\right)}(t) e^{-\alpha t} d t\right] \\
& = \begin{cases}\sum_{\left(i, m_{i}\right) \in S}\left(\frac{1}{\alpha} p_{i}-\frac{1}{\alpha^{2}}\left(1-\phi_{i}(\alpha)\right) E_{u}\left[e^{\left.\left.-\alpha t_{\left(i, m_{i}\right)}\right]\right)}\right.\right. & \text { if } \alpha>0 \\
\sum_{\left(i, m_{i}\right) \in S}\left(p_{i} E_{u}\left[t_{\left(i, m_{i}\right)}\right]+\frac{1}{2}\left(p_{i}^{2}+\sigma_{i}^{2}\right)\right) & \text { if } \alpha=0\end{cases} \\
& = \begin{cases}\sum_{\left(i, m_{i}\right) \in S} A_{\left(i, m_{i}\right)}^{S_{i}, \alpha} x_{\left(i, m_{i}\right)}^{u, \alpha}+\sum_{i \in N}\left|S_{i}\right|\left(\frac{1}{\alpha} p_{i}-\frac{1}{\alpha^{2}} \frac{1-\phi_{i}(\alpha)}{\phi_{i}(\alpha)}\right) & \text { if } \alpha>0 ; \\
\sum_{\left(i, m_{i}\right) \in S}^{S_{i}, 0} A_{\left(i, m_{i}\right)}^{S_{i}} x_{\left(i, m_{i}\right)}^{u, 0}+\sum_{i \in N}\left|S_{i}\right|\left(\frac{1}{2}\left(\sigma_{i}^{2}-p_{i}^{2}\right)\right) & \text { if } \alpha=0 .\end{cases}
\end{aligned}
$$

Now, combining (24) and (26) yields, after straightforward simplification, the required result.

(b) This corresponds to the special case where $S=\widehat{N}$ in part (a).

Note that it follows directly from Theorem 3 that problem $(6$ is an LP relaxation of the scheduling problem (3). Furthermore, application of the PCL framework reviewed in Section 2 yields now the following results: satisfaction of the PCL in Theorem 3 implies that, under the range of admissible holding cost coefficients defined in Section 2,

(1) problem (6) is an exact LP formulation of the scheduling problem (in that their optimal values coincide); and

(2) there exist indices $\gamma_{\left(i, m_{i}\right)}^{\alpha}$ defined for each expanded class, such that it is optimal for problem (3) to work at each time on a job whose current index $\gamma_{\left(i, m_{i}\right)}$ (which depends on its expanded class) is larger.

Furthermore, in addition to satisfying PCL, we see that the conditions for the index decomposition property reviewed in Section 2.1 hold. The projects referred to in that section correspond naturally in the model under study to the original job classes $i \in N$ : the full expanded class set $\widehat{N}$ is thus partitioned into expanded class subsets

$$
\widehat{N}_{i}=\left\{\left(i, m_{i}\right): 1 \leq m_{i} \leq m_{i}^{0}\right\}, \quad i \in N .
$$

It is then immediate to check that, taking the corresponding family of feasible expanded class sets $\mathcal{F}_{i}$ for each class $i$, defined in (5), and using (18), Assumption 3 holds. Therefore, Theorem 2 applies, and it follows that the indices $\gamma_{\left(i, m_{i}\right)}$ can be computed separately for each original class $i$ (i.e., they are decomposable with respect to $i$ ).

\section{Index computation and cost admissibility}

As argued in the previous section, the indices $\gamma_{\left(i, m_{i}\right)}^{\alpha}$ corresponding to the PCL in Theorem 3 are computed separately for each job class $i$. Therefore (cf. Section 2.1), the indices $\gamma_{\left(i, m_{i}\right)}^{\alpha}$ corresponding to a given class $i$ are obtained by applying the adaptive-greedy algorithm $\operatorname{AG}\left(\cdot \mid \mathcal{F}_{i}\right)$ on the input costs $C_{\left(i, m_{i}\right)}$, for $1 \leq m_{i} \leq m_{i}^{0}$. The resulting indices $\gamma_{\left(i, m_{i}\right)}^{\alpha}$, as well as 
the range of admissible costs defined by condition (15), under which the scheduling problem of concern is $P C L$-indexable with respect to the family of $\mathcal{F}$-policies (see Definition 2) are stated next.

Theorem 4 (PCL-indexability) Under Assumption 1 on cost coefficients, scheduling problem (3) is PCL-indexable with respect to the family of $\mathcal{F}$-policies. The optimal priority indices are as follows:

(a) in the discounted case,

$$
\gamma_{\left(i, m_{i}\right)}^{\alpha}=\frac{\alpha \phi_{i}(\alpha)}{1-\phi_{i}(\alpha)} \Delta C_{\left(i, m_{i}\right)}, \quad\left(i, m_{i}\right) \in \widehat{N}
$$

(b) in the undiscounted case $(\alpha=0)$,

$$
\gamma_{\left(i, m_{i}\right)}^{0}=\frac{\Delta C_{\left(i, m_{i}\right)}}{p_{i}}, \quad\left(i, m_{i}\right) \in \widehat{N} .
$$

\section{Proof}

(a) Application of algorithm $\mathrm{AG}\left(\cdot \mid \mathcal{F}_{i}\right)$ to input $\operatorname{costs} C_{\left(i, m_{i}\right)}$, for $1 \leq m_{i} \leq m_{i}^{0}$ yields a set of indices $\gamma_{\left(i, m_{i}\right)}^{\alpha}$ defined as follows:

$$
\gamma_{(i, 1)}^{\alpha}=\frac{C_{(i, 1)}}{A_{(i, 1)}^{\left\{(i, 1), \ldots,\left(i, m_{i}^{0}\right)\right\}, \alpha}}
$$

and

$$
\gamma_{\left(i, m_{i}\right)}^{\alpha}=\gamma_{\left(i, m_{i}-1\right)}^{\alpha}+\frac{C_{\left(i, m_{i}\right)}-\sum_{k_{i}=1}^{m_{i}-1} A_{\left(i, m_{i}\right)}^{\left\{\left(i, k_{i}\right), \ldots,\left(i, m_{i}^{0}\right)\right\}, \alpha}\left(\gamma_{\left(i, k_{i}\right)}^{\alpha}-\gamma_{\left(i, k_{i}-1\right)}^{\alpha}\right)}{A_{\left(i, m_{i}\right)}^{\left\{\left(i, m_{i}\right), \ldots,\left(i, m_{i}^{0}\right)\right\}, \alpha}}, \quad 2 \leq m_{i} \leq m_{i}^{0},
$$

where we write (for notational convenience) $\gamma_{(i, 0)}^{\alpha}=0$. Now, straightforward algebraic simplification of (27) and (28) using (19) yields the expressions for the indices stated in parts (a) and (b) of the Theorem.

Furthermore, the general conditions (15) defining the range of admissible costs are, in this case,

$$
0 \leq \gamma_{(i, 1)}^{\alpha} \leq \cdots \leq \gamma_{\left(i, m_{i}^{0}\right)}^{\alpha}
$$

In light of the expressions in (a), (b) for the indices $\gamma_{\left(i, m_{i}\right)}^{\alpha},(29)$ is equivalent to

$$
0 \leq \Delta C_{(i, 1)} \leq \cdots \leq \Delta C_{\left(i, m_{i}^{0}\right)}
$$

which completes the proof.

\section{References}

[1] Bertsimas, D. and Niño-Mora, J. (1996). Conservation laws, extended polymatroids and multiarmed bandit problems; a unified approach to indexable systems. Math. Oper. Res. 21 257-306. 
[2] Cox, D. R. and Smith, W. L. (1961). Queues. Methuen, London.

[3] Dacre, M., Glazebrook, K.D. and Niño-Mora, J. (1999). The achievable region approach to the optimal control of stochastic systems (with discussion). J. Roy. Statist. Soc. Ser. B 61 747-791.

[4] Edmonds, J. (1970). Submodular functions, matroids, and certain polyhedra. In Combinatorial Structures and their Applications (Proc. Calgary Internat. Conf., Calgary, Alta., 1969), 69-87, Gordon and Breach, New York.

[5] Gittins, J. C. and Jones, D. M. (1974). A dynamic allocation index for the sequential design of experiments. In Progress in Statistics (European Meeting of Statisticians, Budapest, 1972), pp. 241-266. Colloq. Math. Soc. Janos Bolyai, Vol. 9, North-Holland, Amsterdam.

[6] Gittins, J. C. (1979). Bandit processes and dynamic allocation indices (with discussion). J. Roy. Statist. Soc. Ser. B 41 148-177.

[7] Harrison, J. M. (1975). Dynamic scheduling of a multiclass queue: Discount optimality. Oper. Res. 23 270-282.

[8] Klimov, G.P. (1974). Time sharing service systems I. Theory Probab. Appl. 19 532-551.

[9] Korte, B. and Lovász, L. (1981). Mathematical structures underlying greedy algorithms. In Fundamentals of Computation Theory, Lecture Notes in Computer Science, vol. 117, F. Grecseg (Ed.), Springer, Berlin-New York, pp. 205-209.

[10] Meilijson, I. and Weiss, G. (1977). Multiple feedback at a single-server station. Stochastic Process. Appl. 5 195-205.

[11] Niño-Mora, J. (1999). Restless bandits, partial conservation laws, and indexability. Forthcoming in Adv. in Appl. Probab. vol. 33 no. 1 (March 2001).

[12] Niño-Mora, J. (2000). On certain greedoid polyhedra, partially indexable scheduling problems, and extended restless bandit allocation indices. Working Paper Ref. 456, Dept. of Economics and Business, Universitat Pompeu Fabra. Available at: http://www.econ.upf.es/cgi-bin/onepaper?456. Submitted to Mathematical Programming.

[13] Queyranne, M. (1993). Structure of a simple scheduling polyhedron. Math. Programming $58263-285$.

[14] Righter, R. and Xu, S. H. (1991). Scheduling jobs on nonidentical IFR processors to minimize general cost functions. Adv. in Appl. Probab. 23 909-924. 
[15] Rothblum, U. G. and Rothkopf, M. H. (1994). Dynamic recomputation cannot extend the optimality range of priority indices. Oper. Res. 42 669-676.

[16] Rothkopf, M. H. (1966). Scheduling with random service times. Management Sci. 12 707-713.

[17] Rothkopf, M. H. and Smith, S. A. (1984). There are no undiscovered priority index sequencing rules for minimizing total delay costs. Oper. Res. 32 451-456.

[18] Smith, W. E. (1956). Various optimizers for single-stage production. Naval Res. Logist. Quart. 3 59-66.

[19] Tcha, D. W. and Pliska, S. R. (1977). Optimal control of single-server queueing networks and multiclass $M / G / 1$ queues with feedback. Oper. Res. 25 248-258.

[20] van Mieghem, J. A. (1995). Dynamic scheduling with convex delay costs: the generalized cu rule. Ann. Appl. Probab. 5 809-833.

[21] Weber, R. R. (1988). Stochastic scheduling on parallel processors and minimization of concave functions of completion times. In Stochastic differential systems, stochastic control theory and applications (Minneapolis, Minn., 1986), pp. 601-609, IMA Vol. Math. Appl., 10, Springer, New York-Berlin.

[22] Weiss, G. (1988). Branching bandit processes. Probab. Engrg. Inform. Sci. 2 269-278. 